\title{
A multicarotenoid beadlet for human nutrition - proof of concept of in vitro timed release*
}

\author{
Kevin Gellenbeck ${ }^{\boxplus}$, Dawna Salter-Venzon², Rajendra Lala³ and Jayanthi Chavan³ \\ ${ }^{1}$ Nutrilite Health Institute, Lakeview, California, USA; ${ }^{2}$ Nutrilite Health Institute, Buena Park, California, USA; ${ }^{3}$ Omniactive Health Technologies, \\ Wagle Industrial Estate, Thane, India
}

Since the 1980's when the predominate focus of study and use of carotenoids in human nutritional formulations was solely on beta-carotene, there has been a steady increase in research aimed to understand the role of a wide variety of carotenoids in human health. This work has increasingly demonstrated the benefits of a number of carotenoids, and there has been a corresponding increase in the number of carotenoids provided in nutritional supplements (multicarotenoids). Numerous published observations in both human and animal studies suggest significant interaction and competition between various carotenoids during absorption and metabolism, resulting in the inhibition of uptake of one over the other. This competition has the end result of reducing the beneficial effects of the inhibited carotenoid. To limit such competition and maximize carotenoid uptakes, a layered beadlet was designed to release a defined ratio of carotenoids sequentially. Preliminary dissolution testing is presented showing the release profile in simulated digestive conditions of a combination of betacarotene, alpha carotene, lutein, zeaxanthin, lycopene and astaxanthin derived from natural sources. Comparison is made to an immediate release beadlet formulation using the same combination of carotenoids. These results will be used to guide proof of concept clinical testing for effectiveness in humans.

Key words: carotenoid, controlled release, dissolution, bioavailability

Received: 13 October, 2011; accepted: 01March, 2012;

available on-line: 17 March, 2012

\section{INTRODUCTION}

The results of epidemiological studies over the last 30 years consistently demonstrate an inverse relationship between carotenoid-rich fruits and vegetable consumption and risk of chronic disease (Mayne, 1996), but human dietary intakes of fruit and vegetables are repeatedly found to be inadequate for optimal health (WHO technical report, 2002; Blanck et al., 2008). Therefore, it may be useful to provide the consumer a balanced and mixed carotenoid supplement to address this deficit. Supraphysiological doses of individual carotenoids have shown unexpected and worrisome results in the past, as in the case of $\beta$-carotene (Omenn et al., 1996). Such results indicate the need for special attention to be given to supplementation that approximates intakes found in healthy diets with nutritional amounts of naturally derived carotenoids.

A key factor in the efficacy of a mixed carotenoid supplement is the fraction of the ingested amount that is absorbed and reaches its target site(s). While the details remain unclear, much evidence suggests individual carotenoids compete with one another for micelle formation and intestinal absorption (van den Berg et al., 1998; 1999; van het Hof et al., 2000; Maiani et al., 2009). The notion of a supplement designed to provide a balanced carotenoid mix that separates temporally the individual carotenoids within the intestinal tract is compelling for its potential to minimize competition, improve uptake, and maximize efficacy.

In this study we designed and tested the dissolution of a combination carotenoid formulation that A) provides a mix of natural carotenoids in a balanced and optimized ratio and B) incorporates a sequential-release of individual carotenoids across gut transit time of approximately 6 hours.

The carotenoids found in plasma that are associated with a variety of fruit and vegetable intake are $\beta$-carotene, $\alpha$-carotene, lutein, lycopene, and zeaxanthin (Maiani et al., 2009). We included each of these carotenoids, as well as astaxanthin because of its well-known health benefits and the fact that very few diets incorporate food sources rich in this nutrient (di Mascio et al., 1991). We determined our carotenoid ratio by incorporating key aspects of well-accepted dietary guidelines, and considered typical and wide-spread dietary inadequacies or imbalances, such as those exposed in the results from America's Phytonutrient Report (Nutrilite Health Institute, 2010), a study which examined the food consumption records collected as part of the USA National Health and Nutrition Examination Survey (NHANES). America's Phytonutrient Report provided a carotenoid intake amount that would be found in a basic "prudent diet", a diet that provides at least 5 servings of fruit and vegetables per day. The amount and balance of carotenoids found in a "prudent diet" served as our baseline to create an optimized ratio of carotenoids that, when consumed, would help convert an unbalanced and inadequate carotene intake of a "typical" diet into a more balanced and adequate amount, closer to what would be obtained from eating the more healthy "prudent diet". Notably, the ratio was not designed to simply increase the amounts of carotenoids but rather to promote a balanced intake level of mixed carotenoids. For example, the "typical" American diet has a remarkably heavy usage of tomato-based sauces providing for higher lycopene intake in relation to other important carotenoids. Thus, since a "typical" diet is heavier in lycopene, we chose to supplement with a lower level of lycopene, in

e-mail Kevin.Gellenbeck@Amway.com

*Presented at the 16th International Symposium on Carotenoids, 17-22 July, 2011, Kraków, Poland

Abbreviations: CD, controlled release; IR, immediate release; RSD, relative standard deviation. 
Table 1. Carotenoids and ratios included in mixed carotenoid beadlet formulation.

The ratio of carotenoids was established by evaluating the carotenoid levels between a) a diet that meets dietary guidelines for optimal health, and b) a typical consumer's diet. The difference and imbalances between the carotenoid amounts of these two diets were used to create a ratio that will provide a balanced carotenoid intake that closely aligns to what would be found in a recommended prudent diet of at least 5 servings of yellow and green-pigmented fruits and vegetables per day.

\begin{tabular}{lccc}
\hline Carotenoid & $\begin{array}{c}\text { Suggested daily } \\
\text { intake }(\mathrm{mg})\end{array}$ & Selected ratio & $\begin{array}{l}\text { Release profi- } \\
\text { le (hours) }\end{array}$ \\
\hline -carotene & 6 & 12 & $4-6$ \\
a-carotene & 1 & 2 & $4-6$ \\
Lycopene & 2 & 4 & $3-4$ \\
Lutein & 3 & 6 & $2-3$ \\
Zeaxanthin & 0.5 & 1 & $2-3$ \\
Astaxanthin & 0.5 & 1 & $1-2$ \\
\hline
\end{tabular}

order to keep the ratio between the individual carotenoids balanced, so a "typical" user will receive a carotenoids in a ratio of what is estimated to be found in the recommended "prudent diet" containing at least 5 servings of red, yellow, and green-pigmented fruits and vegetables per day. This ratio is shown in Table 1.

Supplementation with synchronous carotenoids presents the challenge of competition with one another for uptake within the enteric system. There is much interaction between various individual carotenoids during micelle formation and perhaps during receptor mediated uptake resulting in the inhibition of uptake of one over the other (van het Hof et al., 2000; Maiani et al., 2009). To mitigate such competition and potentially maximize uptake of individual carotenoids from a multicarotenoid formulation, we have designed a layered beadlet that sequentially releases individual carotenoids over the transit time of the upper intestinal system of the human gut (6 hours). The sequence of release was designed by taking into consideration the known interactions between specific carotenoids and evidence suggesting when each carotenoid might first appear in the triacylglycerol rich fraction of plasma after oral administration (Cardinault et al., 2003; Bierer et al., 1995; van den Berg et al., 1999; Zaripheh \& Erdman, 2002; Johnson et al., 1997; O’Neill \& Thurnham, 1997; Tyssandier et al., 2002; van het Hof et al., 2000). This sequence and proposed dissolution timing is indicated in Table 1.

\section{MATERIALS AND METHODS}

Three multicarotenoid prototypes were prepared by Omniactive Health Technologies using proprietary technology. The controlled release formulations are described in Fig. 1. An inner sugar core has layers of carotenoid extracts applied along with functional release coats and an outer protective coat. The final form is a small beadlet suitable for inclusion in tablet formulations. In addition, an immediate release prototype formulation was prepared for comparative experimentation. This beadlet had a very similar content of the carotenoid extracts as the controlled release beadlet, but in this case only the protective outer coat was applied. Once this layer dissolves, all the contents are intended to release at the same time.

Dissolution tests. Each prototype was subjected to a dissolution test designed to observe the release of carot-

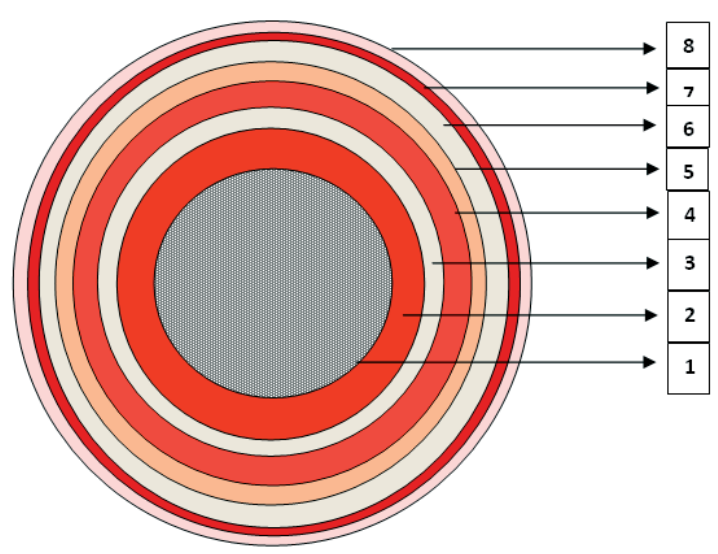

Figure 1. Controlled Release Beadlet Design-Carotenoid.

Extracts from the indicated natural sources are sequentially layered on the inner core and two inner functional coats and one outer protective coat (proprietary) are added to control release in the digestive tract over the indicated time period of 6 hours. (1) NPS/Sugar Sphere (\#60-80); (2) Beta carotene (Dunaliella algae, BASF Corp.) \& Alpha carotene (palm, Chr Hansen Corp.); (3) Functional coat I; (4) Lycopene (tomato); (5) Lutein \& Zeaxanthin (marigold); (6) Functional coat II; (7) Astaxanthin (Haematococcus algae, Alga Technologies); (8) Protective coat III.

enoids into the medium over a 6 hour time period simulating passage through the digestive system. The dissolution methodology was developed in-house at Omniactive Health Technologies based on a review of the US FDA (2011) access data for dissolution with consideration of the physio-chemical parameters of the carotenoids, particularly the limited solubility in aqueous solutions. The details of this process are given in Table 2.

The dissolution test is initiated in the acid stage and the beadlets are added to the stirred vessels in a constant temperature water bath protected from light. At each sampling interval a $20 \mathrm{ml}$ aliquot is removed and replaced with $20 \mathrm{ml}$ of fresh $0.1 \mathrm{~N} \mathrm{HCl}$ solution (add 8.5 $\mathrm{ml}$ of conc. $\mathrm{HCl}$ to $1000 \mathrm{ml}$ demineralized water). The sample is filtered with a 100 mesh ASTM stainless steel sieve to remove undissolved beadlet material into a 50 $\mathrm{ml}$ centrifuge tube and $10 \mathrm{ml}$ of ethyl acetate and $2 \mathrm{~g}$ of sodium chloride are added and the mixture shaken for 30 minutes and centrifuged at $3000 \mathrm{rpm}$ for 5 minutes. $1.5 \mathrm{ml}$ of the upper organic layer is removed and made

Table 2. Parameters used for dissolution testing

\begin{tabular}{|c|c|}
\hline $\begin{array}{l}\text { Dissolution medium vo- } \\
\text { lume }\end{array}$ & $500 \mathrm{ml}$ \\
\hline Type & $\begin{array}{l}\text { USP Type II Paddle } \\
\text { (Model TDT-08L from Electrolab, } \\
\text { Mumbai, Maharashtra, India) }\end{array}$ \\
\hline RPM & 100 \\
\hline Temperature & $37^{\circ} \mathrm{C} \pm 0.5^{\circ} \mathrm{C}$ \\
\hline Time Intervals & $\begin{array}{l}2 \text { hrs in } 0.1 \mathrm{~N} \mathrm{HCl} \text {, continued with } \\
6 \text { hrs in pH } 6.8 \text { buffer with } 1.5 \% \\
\text { SLS (Sodium Lauryl Sulfate) }\end{array}$ \\
\hline Sampling time & Hourly for 8 hours \\
\hline Aliquots volume & $20 \mathrm{ml}$ \\
\hline Aliquots replenish volume & $20 \mathrm{ml}$ \\
\hline Beadlet quantity & $700 \mathrm{mg}$ \\
\hline
\end{tabular}


up to volume, filtered through a $0.45 \mu \mathrm{m}$ nylon syringe filter and injected for HPLC analysis. After 2 hours the $0.1 \mathrm{~N} \mathrm{HC}$ solution is drained from the dissolution vessels and replaced with $500 \mathrm{ml}$ of a $\mathrm{pH} 6.8$ phosphate buffer (Add $6.8 \mathrm{gm}$ of potassium dihydrogen phosphate, $15 \mathrm{~g}$ sodium lauryl sulfate and $0.89 \mathrm{~g}$ of sodium hydroxide in $1000 \mathrm{ml}$ of demineralized water and adjust to $\mathrm{pH}$ 6.8 with sodium hydroxide solution). As before, $20 \mathrm{ml}$ aliquots are removed at the designated sampling intervals and replaced with $20 \mathrm{ml}$ of fresh $\mathrm{pH} 6.8$ phosphate buffer and processed for HPLC analysis.

HPLC analysis was carried out using a Waters Separation Module Alliance 2695 with a 2990 detector according to the following methods and conditions for lutein, zeaxanthin, beta-carotene, alpha-carotene and lycopene:

- All standards used were from Chromadex:

1. Lutein, Cat\#ASB-00012453-5MG, Lot\#000124530549, $95.90 \%$ purity.

2. Beta Carotene, Cat\#ASB-00003211-5MG, Lot\# 00003211-316, $90.20 \%$ purity.

3. Lycopene, Cat\#ASB-00012550-5MG，Lot\#0012550$171,88.40 \%$ purity.

4. Alpha Carotene, Cat\#ASB 00003204-1MG, Lot\# 00003204-10A, 85.60\% purity.

- Column: YMC Carotenoid (C-30), $5.0 \mu \mathrm{m}, 4.6 \times 250$ $\mathrm{mm}$

- Mobile Phase: Channel C: 80/20 Methanol/Chloroform, Channel D: 50/50 Methanol/Chloroform

- Flow Rate: $1.5 \mathrm{~mL} / \mathrm{min}$, Injection volume: $10 \mu \mathrm{L}$, Run time 50 min., Column temperature $30^{\circ} \mathrm{C}$

- Analytical Wavelengths: $474 \mathrm{~nm}$

- Gradient Table:

$\begin{array}{ccc}\text { Time } & \% \mathrm{C} & \% \mathrm{D} \\ 0.0 & 100 & 0 \\ 20.0 & 100 & 0 \\ 32.0 & 100 & 0 \\ 32.1 & 0 & 100 \\ 40.0 & 0 & 100 \\ 40.1 & 100 & 0 \\ 50.0 & 100 & 0\end{array}$

Astaxanthin analysis required a different method and was carried out as follows:

- Standard: Astaxanthin, Chromadex, Cat\#SB-000016972.5ML, Lot\#00001697-102, 96.0\% purity.

- Column: YMC Carotenoids (C-30), $5.0 \mu \mathrm{m}, 4.6 \times 250$ $\mathrm{mm}$

- Mobile Phase: Channel A: 100\% Methanol, Channel B: 100\% MTBE (t-butyl-methyl-ether) — for low loads, Channel C: 1\% phosphoric acid — for high loads

- Flow Rate: $1.0 \mathrm{~mL} / \mathrm{min}$, Injection: $20 \mu \mathrm{L}$, Run time 35 min., Column Temperature $25^{\circ} \mathrm{C}$

- Analytical Wavelength: $274 \mathrm{~nm}$

- Gradient Table:

$\begin{array}{cccc}\text { Time } & \% \mathrm{~A} & \% \mathrm{~B} & \% \mathrm{C} \\ 0.0 & 81 & 15 & 4 \\ 15.0 & 66 & 30 & 4 \\ 23.0 & 16 & 80 & 4 \\ 27.1 & 16 & 80 & 4 \\ 27.1 & 81 & 15 & 4 \\ 35.0 & 81 & 15 & 4\end{array}$

\section{RESULTS AND DISCUSSION}

The carotenoid composition of the three multicarotenoids beadlet formulations is given in Table 3 . The total carotenoid loading in the Immediate Release formulation was about $7.7 \%$ while the controlled release contained about $4 \%$ due to the additional functional coating volume required in the inner layers of the beadlet.

The release profiles over the 6 hour period of the dissolution tests are presented for each of the prototypes in Figs. 2, 3 and 4. The dissolution study was performed in 6 different units and the data was found to have an RSD of $<6 \%$. The Immediate Release formulation in Fig. 2 performed as expected and within 1 hour essentially all the carotenoid content was released to the dissolution medium. In an in vivo digestive system the competitive uptake interactions described in the introduction would be demonstrated and we theorize that overall uptake and

Table 3. Composition of Immediate Release (IR) and Controlled Release (CR) Beadlets (mg/g).

\begin{tabular}{llll}
\hline & $\begin{array}{l}\text { IR Proto- } \\
\text { type }\end{array}$ & $\begin{array}{l}\text { CR Proto- } \\
\text { type 1 }\end{array}$ & $\begin{array}{l}\text { CR Proto- } \\
\text { type 2 }\end{array}$ \\
\hline Beta carotene & 34.30 & 16.34 & 18.83 \\
Lutein & 20.73 & 8.80 & 10.52 \\
Lycopene & 10.49 & 5.83 & 5.34 \\
Alpha carotene & 4.79 & 3.10 & 3.35 \\
Zeaxanthin & 5.13 & 2.52 & 2.28 \\
Astaxanthin & 1.57 & 1.50 & 1.53 \\
\hline
\end{tabular}

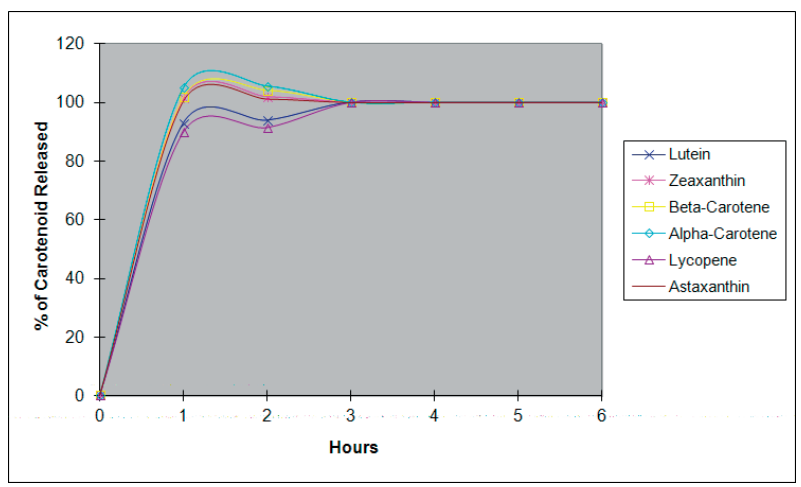

Figure 2. Dissolution profile of the Immediate Release Prototype expressed as the percentage of the original beadlet load of each carotenoid released to the dissolution medium over time.

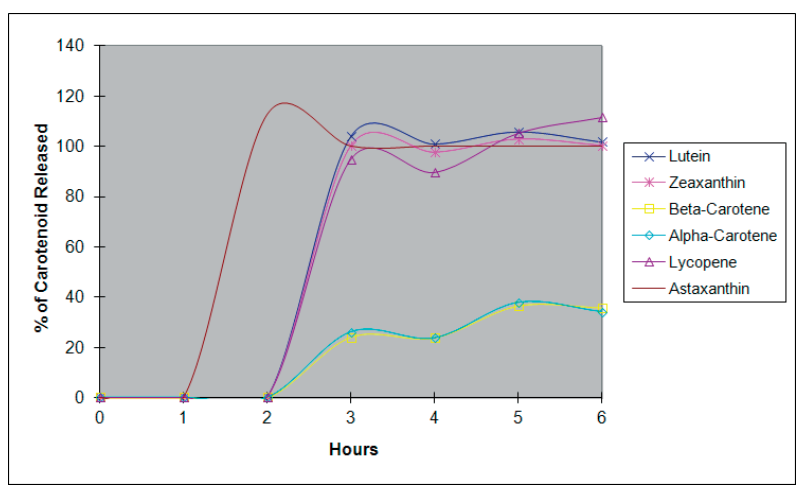

Figure 3. Dissolution profile of the Controlled Release Prototype I expressed as the percentage of the original beadlet load of each carotenoid released to the dissolution medium over time. 


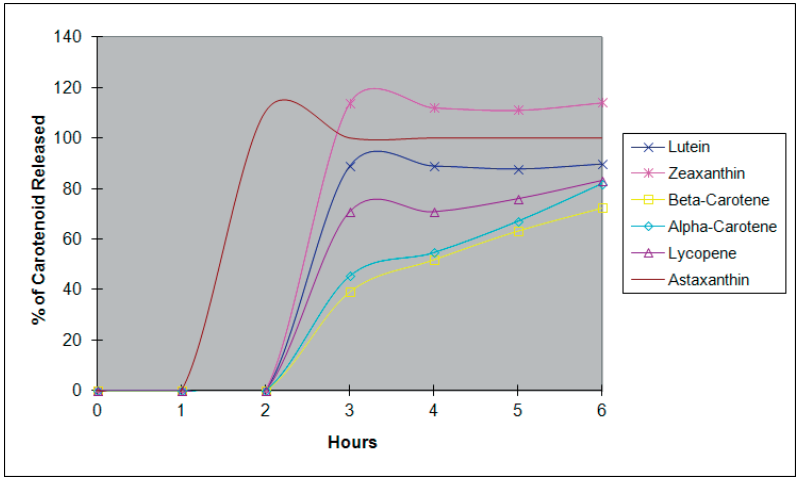

Figure 4. Dissolution profile of the Controlled Release Prototype II expressed as the percentage of the original beadlet load of each carotenoid released to the dissolution medium over time.

bioavailability in the body would be reduced from what would be expected for the same concentrations provided individually.

The Controlled Release I Prototype in Fig. 3 released the astaxanthin immediately and then lycopene, lutein and zeaxanthin very quickly after the dissolution medium was changed to the higher $\mathrm{pH}$, but the beta-carotene and alpha-carotene were resistant to release and after the total 6 hours only released about $40 \%$ of the available total. Modifications were made in the composition of the release retarding polymers in the second Controlled Release Prototype in Fig. 4. This resulted in a similar complete release of the astaxanthin, lycopene, lutein and zeaxanthin, and an improved release of the beta-carotene and alpha-carotene that neared complete release at the end of the 6 hour experimental period.

These preliminary dissolution experiments demonstrate the ability to provide significant separation of carotenoid release sequentially from a single beadlet preparation. Modifications in the preparation were shown to change the release profile and future formulations will attempt to further separate carotenoids during dissolution and to provide complete release within the 6 hour time period characteristic of maximal uptake in the human digestive system.

Once the beadlet formulation is optimized, the dissolution test data will be used to support a full clinical comparison of the immediate and controlled release beadlets. The in vivo situation will be used to fully test the hypothesis that a controlled release formulation will improve the bioavailability of carotenoids for human nutrition.

\section{Acknowledgements}

From Amway Corporation, substantial contributions to this work were provided by Amitabh Chandra and Nathan Stern on the analytical chemistry and Janjira Intra on the dissolution evaluation. From Omniactive Health Technologies, Jayant Deshpande provided substantial contribution on the beadlet production and dissolution testing. Their assistance is greatly appreciated.

\section{REFERENCES}

Bierer TL, Merchen NR, Erdman JW (1995) Comparative absorption and transport of five common carotenoids in preruminant calves. $J$ Nutr 125: 1569-1577.

Blanck HM, Gillespie C, Kimmons JE, Seymour JD, Serdula MK (2008) Trends in fruit and vegetable consumption among US men and women, 1994-2005. Prev Chronic Dis 5: 2. http://www.cdc. gove/pcd/issues/2008/apr/07_0049.htm.

Cardinault N, Tyssandier V, Grolier P, Winklhofer-Roob B, Ribalta J, Bouteloup-Demange C, Rock E, Borel P (2003) Comparison of the postprandial chlymicron carotenoid responses in young and older subjects. Eur I Nutr 42: 315-323.

di Mascio P, Murphy ME, Sies H (1991) Antioxidant defense systems: The role of carotenoids, tocopherols and thiols. Am J Clin Nutr 53: 194S-200S.

Johnson EJ, Qin J, Krinsky NI, Russell RM (1997) Ingestion by men of a combined dose of beta-carotene and lycopene does not affect the absorption of beta-carotene but improves that of lycopene. $J$ Nutr 127: 1833-1837.

Maiani G, Caston MJP, Catasta G, Toti E, Cambrodon IG, Bysted A, Granada-Lorencio F, Olmedilla-Alonso B, Knuthesen P, Valoti M, Bohm V, Maver-Mieback E, Behsnilian D, Schlemmer U (2009) Carotenoids: Actual knowledge of food sources, intakes, stability and bioavailability and their protective role in humans. Mol Nutr Food Res 53: S194-S218.

Mayne ST (1996) Beta-Carotene, carotenoids and disease prevention in humans. FASEB J 10: 690-701.

Nutrilite Health Institute (2010) America's Phytonutrient Report: Quantifying the Gap. http://www.nutrilite.com/en-us/Media/ AmericaPhytonutrientReport.pdf

Omenn GS, Goodman GE, Thornquist MD, Balmes J, Cullen MR, Glass A, Keogh JP, Meyskens FL Jr, Valanis B, Williams JH Jr, Barnhart S, Cherniack MG, Brodkin CA, Hammar S (1996) Effects of a combination of beta carotene and vitamin $A$ on lung cancer and cardiovascular disease. N Engl J Med 334: 1150-1155.

O’Neill ME, Thurnham DI (1998) Intestinal absorption of beta-carotene, lycopene and lutein in men and women following a standard meal: response curves in the triacylglycerol-rich lipoprotein fraction. Brit J Nutr 79: 149-159.

Tysandier V, Cardinault N, Caris-Veyrat C, Amiot M-J, Grolier P, Bouteloup C, Azais-Braesco V, Borel B (2002) Vegetable-borne lutein, lycopene, and beta-carotene compete for incorporation into chylomicrons, with no adverse effect on the medium-term (3-wk) plasma status of carotenoids in humans. Am J Clin Nutr 75: 526534.

US Food and Drug Administration (2011) Dissolution Methods. http://www.accessdata.fda.gov/scripts/cder/dissolution/

WHO/FAQ Expert Consultation Report (2002) Diet, Nutrition and the prevention of chronic disease. WHO technical report series $\mathbf{9 1 6}$. Geneva, Jan 28-Feb 1. http://whqlibdoc.who.int/trs/who_trs_916. pdf

van het Hof KH, West CE, Westerate JA, Hautvast GAJJ (2000) Dietary factors that affect the bioavailability of carotenoids. J Nutr 130: 503-506.

van den Berg H (1998) Effect of lutein on beta-carotene absorption and cleavage Int J Vitam Nutr Res 68: 360-365.

van den Berg H (1999) Carotenoid interactions. Nutr Rev 57:1-10.

Zaripheh S, Erdman JW (2002) Factors that influence the bioavailability of xanthophylls. J Nutr 132: 531S-534S.

\section{Applicable Patents and Applications:}

Omniactive Health Technologies (2002) European Patent EP 1513 804 B1, Australian Patent AU 2002348718 B2, Novel Trans-Lutein Enriched Xanthophyll Ester Concentrate and a Process for its Preparation.

Omniactive Health Technologies (2005) US Patent Pending US 2005/0095301 A1 - Novel Stable Beadlets of Lipophilic Nutrients. Omniactive Health Technologies (2010) Japan Patent Pending 2010159276 - Novel Trans-Lutein Enriched Xanthophyll Ester Concentrate and a Process for its Preparation.

Omniactive Health Technologies (2007) India Patent Pending - Novel Time Release Multi Carotenoid Composition and a Process for its Preparation. Application No: 2181/MUM/2007.

Amway Corporation (2011) US Provisional Patent Application - Multicarotenoid Beadlets, AM1199. 